\title{
Welcome address of the President of the Yamada Science Foundation, Professor Emeritus of Osaka University, Prof. Junjiro Kanamori
}

Published online: 20 March 2012

(C) Springer Science+Business Media B.V. 2012

On behalf of the Yamada Science Foundation, I would like to extend our heartfelt welcome to all participants of the 31st International Conference on the Applications of the Mössbauer Effect (ICME2011). I thank all of you for participating in this conference, overcoming various difficulties caused by the big earthquake of last March. I would like to express also our sincere thanks to Professor Yoshida and other organizers for their extraordinary efforts to prepare and work out this conference.

This conference is supported by the Yamada Science Foundation as the 65th Yamada Conference. I would like to describe briefly the Yamada Science Foundation (YSF), summarizing the outline given in the homepage of the Foundation (http://www.yamadazaidan.jp). The Foundation was established in 1977 with the donation of about 30 million dollars made by late Mr. Kiro Yamada, President of the Rohto Pharmaceutical Company Limited at that time where he worked for more than fifty years. Concerned with the lack of innovative works in Japan's science and technology, he intended to help assist creative basic research in the fields of physics, chemistry and life sciences in Japan. YSF puts emphasis on original and pure science programs by individual scientists that are not always well funded by government and industry. Science has its own intrinsic motivation of development irrespective of whether it is of practical use or not. YSF support is made to promote pure science based on its intrinsic motivation. YSF supports domestic individual research and international conferences and symposia to this purpose. In the selection of support items, an emphasis is laid on interdisciplinary projects. As is described in the objective of this conference, the Mössbauer Spectroscopy is an experimental method which is used in a wide range of research fields of basic sciences, providing with deep insight into the atomistic nature of materials. Thus this conference fits well the objective of the foundation.

Let me introduce myself briefly. I was a professor of theoretical solid state physics at Osaka University from 1965 to 1991 and engaged mainly in the research of magnetism. The Mössbauer effect is naturally one of the subjects which I am interested deeply in. Thus both officially and personally I hope sincerely that this Yamada Conference will become a fruitful event which benefits all participants and will be remembered for a long time. Hoping also that all participants will enjoy the conference and foster friendship, I conclude this welcome address. 